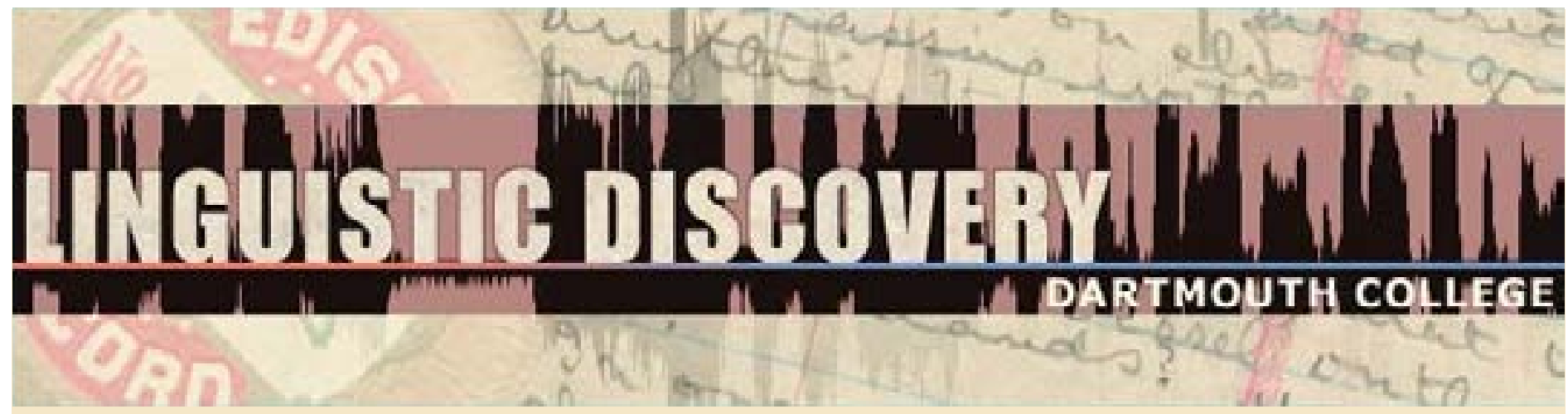

Volume 8 Issue 1 2010

\section{Author's Reply - What Semantic Maps} Show and What They Are Good For

Andrej L. Malchukov

Max Planck Institute for Evolutionary Anthropology, Leipzig

doi: 10.1349/PS1.1537-0852.A.374

url: http://journals.dartmouth.edu/cgi-bin/WebObjects/ Journals.woa/1/xmlpage/1/article/374 


\title{
What Semantic Maps Show and What They Are Good for
}

\section{Author's reply to 'Three Questions about Analyzing Semantic Maps' (Wälchli 2010b) and 'Optimizing Classical Maps' (Narrog 2010)}

\author{
Andrej L. Malchukov \\ Max Planck Institute for Evolutionary Anthropology, Leipzig
}

In their commentaries, Heiko Narrog and Bernhard Wälchli raise interesting questions with the approach I am advocating in my paper. I will start with Bernhard Wälchli's commentary, since it is more on a critical note, and thus requires a more elaborate response.

The main goal of my paper was to question the general assumption commonly underlying semantic maps that recurrent similarities should always reflect semantic affinities; as pointed out, some of the similarities can be attributed to other factors. A secondary goal was to show what consequences this observation has for the semantic map approach. In his comment, Bernhard Wälchli (2010b) does not challenge the actuality of these other factors, but casts doubt on the validity of these findings for the methodology of semantic maps. I will briefly react to the three questions raised by Wälchli here.

\section{Wouldn’t It Be Better to Control for Noise Rather Than Exclude It?}

First, I never meant to say that one should exclude undesirable effects from a semantic map, but rather conflicting factors-I thought the paper was quite explicit in that (see e.g. its concluding section): one should feature out interfering factors (such as zero coding, marked forms of oppositions, etc.) and thus try to get a clearer pattern on the semantic map. In practice, this can be done either through delimiting the domain of investigation from the very beginning, or by coding the relevant factor consistently in the database, so that the impact of this factor can be controlled. For example, one could restrict the investigation from the outset to overt markers (which would also account for violations on the map of case markers discussed in section 6 of my paper), to simplex (one-word) gram markers (which would also account for contiguity violations caused by $t e=i r u$ form discussed in section 6.3), or exclude grammatical categories related to lexical ones (which would also account for the anomalous polysemy pattern of the ma form in Teiwa discussed in section 6.1). ${ }^{1}$ Alternatively, one could control for the impact of these factors through their consistent coding in the dataset (e.g. zero vs. overt markers, simplex vs. complex markers, markers with/without lexical counterparts, etc.). Generally, I would agree with Wälchli that the second approach is preferable, although it is also more time-consuming. In my paper, I argued that one could get a clearer pattern/trend on a semantic map when these interfering factors are controlled for. But in order to do this, such confounding factors first need to be identified, which I did by studying the rare/exceptional polyfunctionality patterns. On the one hand, Wälchli seems to agree with me when he writes about desirability of controlling for the impact of these factors (through the use of statistical methods). On the other hand, however, he writes: "But wouldn't it be better to apply more robust methods for building semantic maps where a little noise does not do any harm?" If this is meant as a suggestion that no further

\footnotetext{
${ }^{1}$ Thus Wälchli (2010a) excludes from consideration local case markers related to (derived from) verbs.
} 
analysis of similarity maps is needed or intended, I tend to disagree. It seems that similarity maps for many domains, if taken at face value, reveal few non-trivial generalizations, and such an approach definitely does not bring us any further in understanding the motivations behind certain patterns (for such an understanding, both general trends as well as exceptions/noise are important, as I argued). Wälchli writes further: "The emerging picture in probabilistic maps is not 'messy' because the method is messy, but rather because this method can be applied to datasets which come closer to reflecting the real amount of diversity in discourse." Clearly, semantic maps are of little use if we just find random variation. They are valuable to the extent to which they visualize certain patterns, and the aim of my article was to suggest how to refine the emerging factors on similarity maps through control of confounding factors.

\section{How Are Preselected Functions Defined?}

The issue of how categories/functions are identified within languages as well as crosslinguistically is a general challenge for linguistic research which shall not be addressed here (but see Haspelmath 2007 for proposals). The selection of functions is subjective and depends ultimately on the focus of investigation. It is conventional to specify ("sharpen") the functions under discussion through the use of examples, which are designed to illustrate the functional properties of the category under consideration. This is especially pressing for less conventional categories (such as Companion in Wälchli 2010a), but it is also important for categories allowing for multiple interpretations. For example, with respect to possessives, one could distinguish between alienable and inalienable possession, further subdivisions may be necessary on syntactic grounds (thus, Heine and Kuteva 2002 distinguish three different syntactic types of possessive constructions). The use of examples is thus a shortcut for such additional functional characteristics. Other than that, it does not have any particular theoretical status in my approach. In this respect, my approach is no different from the use of examples by other authors, such as Haspelmath. This does not mean, of course, that the choice of examples is completely unconstrained. One minimal requirement is that the use of a particular marker should not be idiomatic (i.e. highly lexically restricted). For this reason, an example like This house belongs to John cited by Wälchli would not count as a felicitous example of the possessive use of the preposition, since it expresses possession only in conjunction with a specific verb. (Such idiomatic uses are a frequent source of exceptions/outliers on semantic maps, cf. Wälchli's 2010a discussion of the idiomatic uses of the source preposition $d e$ in the goal function in French).

\section{What Is the Underlying Theory of Similarity?}

I cannot fully follow Wälchli when he writes that "similarity is based on adjacency" in my approach. Rather, similarity is reflected in adjacent ordering as well as through the use of connecting lines, as is also standard in classical semantic maps. Indeed, partial identity of adjacent/connected categories is frequently assumed in the literature (explicitly or implicitly). This overlap is often captured in terms of features (see Zwarts 2010), or, more generally, in terms of some shared semantic components (as in Wierzbicka's chain model of polysemy; e.g. Wierzbicka 1980). I do not think that further decomposition of functions would disturb the map, or that "if adjacency is indispensable for defining similarity, this would mean that the degree of resolution cannot be altered" (Wälchli 2010b). If a certain function is decomposed further (e.g. 
Experiencer into Cognizer, Perceiver, etc.), its sub-varieties share more semantic components with each other than with any other function of the same granularity. If, for example, on a coarse-grained map of syntactic functions, Agent is placed between Experiencer (both share the sentience feature) and Instrument (both are instigating entities), the overall configuration should not change if the roles are decomposed. In some versions of the (classical) semantic map approach, this is explicitly represented by permitting inclusion relations between more general vs. more specific functions on the map. (see van der Auwera and Plungian 1998). Thus, "zooming in" on a particular domain does not change the overall configuration of the semantic map.

Further, Wälchli challenges those approaches to semantic maps which assume (like I do) that semantic similarity involves partial identity. The extent to which such approaches succeed in identifying shared semantic components should be judged on a case-by-case basis; yet I think it raises interesting questions and stimulates semantic analyses to find a common denominator behind different uses of a polyfunctional item. It is less clear to me what the alternative research program of 'similarity semantics' as proposed by Wälchli is. It seems to me that it is content with a statement of different degrees of similarity between particular uses, and stops at this point without posing further research questions. For that reason, even though I find Wälchli's approach intriguing, I have certain reservations about regarding 'similarity semantics' at this stage (whatever its philosophical merits) as a serious competitor to the first approach.

$* * *$

In his otherwise very sympathetic commentary, Heiko Narrog rightfully draws attention to the fact that distinguishability effects differ in some important respects from other interfering factors discussed in my paper. I agree that distinguishability has a special status as a competing motivation; it also stands out as another general functional factor in interaction with semantic similarity, while most other interfering factors are structural, at least in part. As I showed in my paper, distinguishability can induce two kinds of effects. On the one hand, it can yield polysemies reflecting "spurious" semantic connections. This seems to be more common in minimal/highly restricted systems (paradigms). A classical example would be 'morphological reversals' or 'polarity' phenomena (Baerman 2007), where a morphological opposition seems to reverse its function across environments (e.g. the same marker is used for plural of feminine nouns and for the singular of masculines). On the other hand, distinguishability can also inhibit certain polysemies, thus masking a semantic connection (see section 4 of my paper for exemplification). The latter effect seems to be more common and, as I argue in my paper, can explain why certain long-range polysemies do not exist. Indeed, it might be advisable, as also suggested by Narrog, to introduce special conventions for representing the effects of distinguishability (both "spurious connections" as well as "blocking effects") graphically on the map.

\section{References}

Baerman, Matthew. 2007. Morphological reversals. Journal of Linguistics 43/1.33-51. Haspelmath, Martin. 2007. Pre-established categories don't exist: Consequences for language description and typology. Linguistic Typology 11/1.119-132. 
Heine, Bernd and Tania Kuteva. 2002. World lexicon of grammaticalization. Cambridge: Cambridge University Press.

Malchukov, Andrej L. 2010. Analyzing semantic maps: A multifactorial approach. Linguistic Discovery, this issue.

Narrog, Heiko. 2010. Optimizing classical maps. Comment on Malchukov 2010. Linguistic Discovery, this issue.

van der Auwera, Johan and V.A. Plungian. 1998. Modality's semantic map. Linguistic Typology 2/1.125-139.

Wierzbicka, Anna. 1980. The case for surface case. Ann Arbor: Karoma.

Wälchli, Bernhard. 2010a. Similarity semantics and building probabilistic semantic maps from parallel texts. Linguistic Discovery, this issue.

-----. 2010b. Three questions about analyzing semantic maps. Comment on Malchukov 2010. Linguistic Discovery, this issue.

Zwarts, Joost. 2010. Semantic map geometry: Two approaches. Linguistic Discovery, this issue.

Author's contact information:

Andrej L. Malchukov

Department of Linguistics

Max Planck Institute for Evolutionary Anthropology

Deutscher Platz 6

04103 Leipzig

andrej_malchukov@eva.mpg.de 